\title{
DETECTION OF WATER ICE ON THE CENTAUR 1997 CU $_{26}$
}

\author{
Michael E. Brown ${ }^{1}$ And Christopher D. Koresko \\ Division of Geological and Planetary Sciences, California Institute of Technology, Pasadena, CA 91125; \\ mbrown@gps.caltech.edu, koresko@gps.caltech.edu \\ Received 1998 May 5; accepted 1998 July 2; published 1998 August 24
}

\begin{abstract}
We report the detection of the 1.5 and $2.0 \mu \mathrm{m}$ absorption bands due to water ice in the near-infrared reflection spectrum of the Centaur $1997 \mathrm{CU}_{26}$, which is currently located just outside the heliocentric distance of Saturn. The water ice bands are weaker than those detected on the surface of any other solar system body; the spectrum is well fit with a model surface consisting predominantly of a neutral dark absorbing substance with only $~ 3 \%$ areal coverage of water ice. The spectrum thus appears very different from that of the Centaur 5140 Pholus, although both objects are of similar brightness and are at similar heliocentric distances.
\end{abstract}

Subject headings: comets: general — infrared: solar system — minor planets, asteroids

\section{INTRODUCTION}

Near-infrared spectroscopy offers the possibility of determining the surface composition of outer solar system bodies (Brown \& Cruikshank 1997). Such observations have shown the presence of $\mathrm{CH}_{4}, \mathrm{H}_{2} \mathrm{O}$, and $\mathrm{N}_{2}$ ices on Pluto and Triton (Cruikshank et al. 1997), water ice on Charon and on the moons of the giant planets, and potentially organic residues on the Centaur object 5140 Pholus. The surface composition of these objects reflects the initial conditions of the solar nebula at the location at which they were formed as well as any subsequent processing that has taken place since then.

The Kuiper belt objects (KBOs), residing in the relatively cool and quiescent outer solar system beyond Neptune, are thought to be the least processed objects remaining in the solar system, so their composition should most reflect the initial formation conditions. In an attempt to probe these initial conditions, much recent effort has gone into obtaining near-infrared spectra of KBOs (Brown et al. 1997; Luu \& Jewitt 1998). Unfortunately, the signal-to-noise ratios of the spectra obtained have been low because of the extreme faintness of these objects. The KBO 1993 SC was seen to have features possibly due to hydrocarbon ices in a highly smoothed spectrum (Brown et al. 1997), while $1996 \mathrm{TL}_{66}$ appears featureless in a very noisy spectrum (Luu \& Jewitt 1998).

To circumvent the problems associated with the dimness of the KBOs, we are obtaining near-infrared spectra of the brighter Centaur objects. Centaur objects are the presumed transition objects between trans-Neptunian KBOs and short-period comets (see Levison \& Duncan 1997) and are on unstable orbits between the giant planets. Because they have smaller heliocentric distances than KBOs, these objects tend to be brighter, and higher quality spectra can be obtained. Unfortunately, their smaller heliocentric distances also imply larger temperatures, so these objects potentially suffer additional processing beyond that experienced by KBOs. Thus, Centaur object compositions contain information on a combination of the initial abundances and on reactions to heating as the objects move closer to the Sun. Here we present the near-infrared spectrum of the Centaur $1997 \mathrm{CU}_{26}$ and compare it with the spectra of previously observed Centaur objects and KBOs.

\footnotetext{
${ }^{1}$ Alfred P. Sloan Research Fellow.
}

\section{OBSERVATIONS}

Near-infrared spectra of the Centaur $1997 \mathrm{CU}_{26}$ were obtained on 1998 March 7 using the near-infrared camera (Matthews \& Soifer 1994) on the Keck telescope. At the time of observations, the object was 13.7 AU from the Sun, 12.9 AU from the Earth, at a phase angle of 2.4 , and moving at a rate of approximately $6^{\prime \prime} \mathrm{hr}^{-1}$ with respect to the background stars (Minor Planet Electronic Circular S14 [1997]). We identified the object in direct images and centered it in a 0.52 wide spectral slit before inserting the 120 line $\mathrm{mm}^{-1}$ grism and a $\mathrm{H}$ through $K$-band order sorting filter into the light path, which allowed us to collect a spectrum in first order from 1.4 to 2.5 $\mu \mathrm{m}$ at a resolution of approximately $\lambda / \Delta \lambda=100$.

We integrated for a total of $2500 \mathrm{~s}$ on $1997 \mathrm{CU}_{26}$ at airmasses ranging from 1.03 to 1.08 . The spectra were obtained by positioning the object in the center of the long slit, integrating for 200 or $300 \mathrm{~s}$, and then offsetting the telescope $5^{\prime \prime}$ and $10^{\prime \prime}$ north and then south and obtaining similar exposures. We then differenced these individual spectra to remove the sky background and extracted a spectrum at each position. Flat-fielding of the spectra was performed by comparison to spectra of a diffusely illuminated dome spot obtained with identical spectrograph settings. To increase the signal-to-noise ratio of the spectra, we binned by 4 pixels in the spectral dimension for a final wavelength sampling of $0.024 \mu \mathrm{m}$ pixel $^{-1}$.

To correct for telluric absorption, we divided the spectra by the spectrum of a nearby A2 V star (SAO 98558) obtained immediately after the target exposures at an airmass of 1.09. Such a star should show weak narrow hydrogen absorption features from about 1.45 to $1.75 \mu \mathrm{m}$ (Lançon \& Rocca-Volmerange 1992), which could lead to spurious apparent emission features in the divided spectrum at these wavelengths. We then averaged the individual target spectra and multiplied them by the ratio of the blackbody function of the A2 star divided by that of the Sun to obtain a relative reflectance, which we plot in Figure 1. The spectrum shows two approximately $20 \%$ deep broad absorption features centered at 1.5 and $2.0 \mu \mathrm{m}$. Since we were concerned that the slightly higher airmass of the spectral calibrator star could result in spurious features in the divided spectrum, we divided spectra of $1997 \mathrm{CU}_{26}$ obtained at an airmass of 1.03 with those from an airmass of 1.08 , and we determined that no residuals due to such an airmass mismatch were apparent. We thus conclude that the absorption features detected in the spectrum are real. In addition, no apparent emis- 


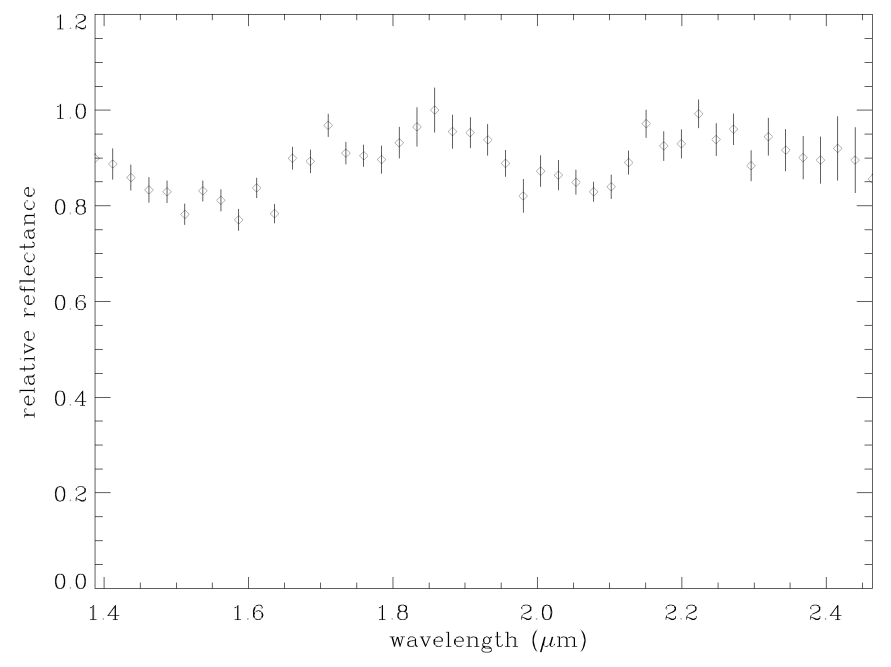

Fig. 1.-Reflectance spectrum of the Centaur object $1997 \mathrm{CU}_{26}$. The spectrum has been scaled to a value of 1.0 at its highest point. The broad absorptions at 1.5 and $2.0 \mu \mathrm{m}$ are characteristic of water ice.

sion lines are visible at the expected locations of the hydrogen absorptions in an $\mathrm{A} 2 \mathrm{~V}$ star.

\section{SPECTRAL MODELING}

The absorption bands detected in $1997 \mathrm{CU}_{26}$ are characteristic of water ice as observed on the satellites of the outer planets. The depth of the absorption bands on $1997 \mathrm{CU}_{26}$ is significantly smaller than those of the bright icy satellites of Jupiter and Saturn and is smaller even than those of the dark icy satellites of Uranus (Brown 1983), which suggests that the surface, like that of the Uranian satellites, is composed primarily of a dark, spectrally neutral material with a small amount of water ice. Recent radiometery of $1997 \mathrm{CU}_{26}$ by Jewitt \& Kalas (1998) confirms that the geometric albedo is less than approximately $5 \%$.

We attempt to fit the infrared spectrum and visual albedo of $1997 \mathrm{CU}_{26}$ using an areal mixture of water ice frost and a spectrally featureless, dark material. Matching the extremely low albedo requires an unusually dark substance; we use the organic material extracted from the Murchison carbonaceous chondrite as a representative dark material (Brown 1983). For water frost, we calculate the reflectance spectrum from the laboratory optical constants of Grundy \& Schmitt (1998) assuming grain sizes of $10 \mu \mathrm{m}$ and an ice temperature of $90 \mathrm{~K}$. The reflectance spectra of these individual components, smoothed to the binned resolution of our spectrum, are shown in Figure 2.

We calculate the wavelength-dependent geometric albedo spectrum of our model mixtures using the formalism of Hapke (1983) assuming isotropic scattering from all grains. We find that the best fit is obtained with a model consisting of 3\% areal coverage of $10 \mu \mathrm{m}$ water ice grains and the remainder the Murchison organic component (Fig. 3). The use of these particular materials for modeling the spectrum of $1997 \mathrm{CU}_{26}$ is somewhat arbitrary; any sufficiently dark, slightly red but spectrally bland component areally mixed with a small amount of water ice can produce a similarly acceptable fit. In addition, as demonstrated by Davies et al. (1997), many different combinations of grain size and ice fraction in both areal and intimate mixtures can be fit to weak water ice absorption bands.

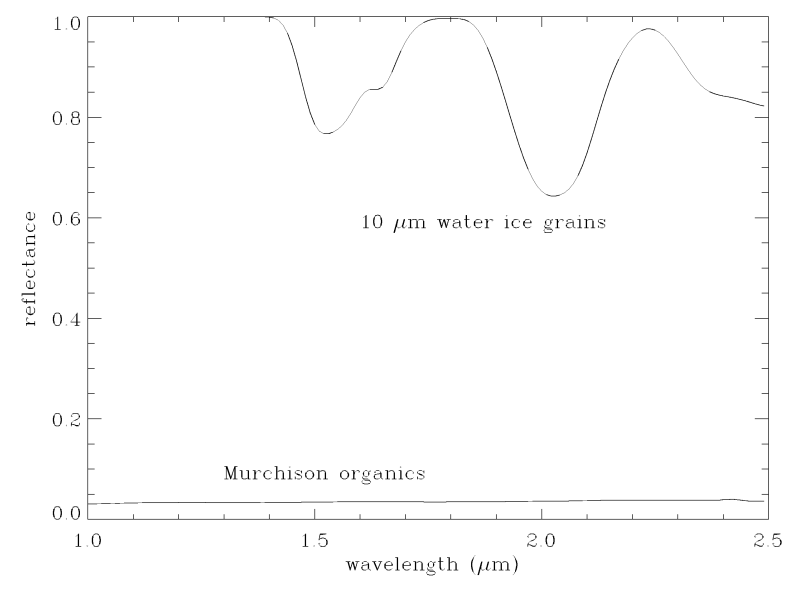

FIG. 2.-Near-infrared reflectance spectra of $10 \mu \mathrm{m}$ water ice grains at $90 \mathrm{~K}$, from the optical constants of Grundy \& Schmitt (1998) water ice frost and of dark organic material extracted from the Murchison carbonaceous chondrite (Brown 1983).

\section{DISCUSSION}

$1997 \mathrm{CU}_{26}$ shows the distinctive near-infrared absorption features of water ice on its surface. Two other Centaur objects, 2060 Chiron and 5145 Pholus, have also had their near-infrared spectra measured (Luu, Jewitt, \& Cloutis 1994; Davies, Sykes, \& Cruikshank 1993), and no two are alike. Chiron shows an essentially featureless spectrum, while Pholus has spectral features reminiscent of ammonia ice and a continuum spectrally similar to "Titan tholin" (Wilson, Saga, \& Thompson 1994), although recent work suggests instead the presence of water ice and complex hydrocarbons (Cruikshank et al. 1998). Spectra of Kuiper belt objects, which are the presumed progenitors of Centaur objects, are equally diverse, with 1993 SC (and Pluto) showing a complex spectrum possibly due to the presence of hydrocarbons (Brown et al. 1997) and $1997 \mathrm{TL}_{66}$ showing an apparently featureless continuum (but note that water ice absorption similar to that seen in $1997 \mathrm{CU}_{26}$ would have been undetectable in the low signal-to-noise ratio spectrum available) (Luu \& Jewitt 1998).

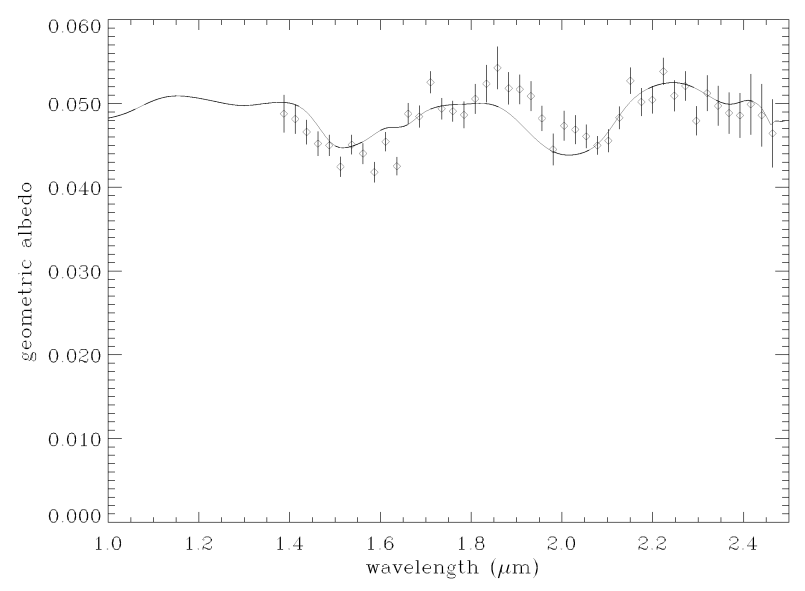

FIG. 3.-Model of the spectrum of $1997 \mathrm{CU}_{26}$ composed of an areal mix of $3 \% 10 \mu \mathrm{m}$ water ice grains and the remainder the Murchison organic material. The measured relative reflectance of $1997 \mathrm{CU}_{26}$ has been scaled to match the model. The spectrum can be equally well fit by a combination of a small amount of water ice and any sufficiently dark, spectrally bland, slightly red material. 
The difference between Chiron and Pholus may be related to Chiron's coma formation (Luu \& Jewitt 1996); Chiron, at a heliocentric distance of almost 9 AU, appears to be sufficiently warm to be active, while Pholus, at more than $13 \mathrm{AU}$, is not. But Pholus and $1997 \mathrm{CU}_{26}$ are at almost identical heliocentric distances, so this simple explanation is insufficient. The two objects do, however, have significantly different perihelia, with Pholus approaching as close as 8.4 AU from the Sun and $1997 \mathrm{CU}_{26}$ approaching no closer than 13.1 AU. In addition, the chaotic nature of Centaur orbits prevents us from accurately knowing their past histories, so the different natures of the two objects could still be due to different heating histories.

Spectroscopic differences between the Centaur objects and KBOs are also potentially explained simply by the greater heating of the Centaur objects leading to the loss of some types of surface ices. Molecular nitrogen ice, which is the dominant surface component on Pluto and Triton (Cruikshank et al. 1997), is unstable at the temperatures of the Centaur objects. $\mathrm{CO}$ and $\mathrm{CH}_{4}$, both also observed on Pluto and Triton, have nonnegligible vapor pressures at Centaur object temperatures and may also be substantially depleted. In addition, the known Centaur objects are smaller than the known KBOs, so gases are quickly lost to space and a frosty surface is harder to develop.

Given these expected reasons for differences among objects, it is surprising to find that distributions of visible colors of Centaur objects and KBOs appear indistinguishable (Luu \& Jewitt 1996) and that both classes of objects even seem to be grouped into the same two distinct color populations in visible light spectrophotometry (Tegler \& Romanishin 1998). We suspect, however, that, since the near-infrared portion of the spectrum is more sensitive to the surface composition, these two distinct populations will not appear as two distinct types of infrared spectra. Indeed, we can already tentatively identify three potential classes of Centaur object and KBO spectra: those dominated by hydrocarbon ice features (1993 SC, Pluto, Pholus), water ice features (1997 $\mathrm{CU}_{26}$ and Charon), and featureless continua (Chiron and $1996 \mathrm{TL}_{66}$ ). Study of the numbers, distributions, and other characteristics of objects in these classes will help to elucidate the mechanisms responsible for the large diversity of surface compositions among and between the Centaur objects and Kuiper belt objects.

Note added in manuscript.-Soon after the submission of this manuscript, Brown et al. (1998) published similar conclusions from an infrared spectrum of $1997 \mathrm{CU}_{26}$ that was obtained 6 months previous to ours.

Data presented herein were obtained at the W. M. Keck Observatory, which is operated as a scientific partnership among the California Institute of Technology, the Universities of California, and the National Aeronautics and Space Administration. The observatory was made possible by the generous financial support of the W. M. Keck Foundation. We thank the referee for useful and informative comments.

\section{REFERENCES}

Brown, R. H. 1983, Icarus, 56, 414

Brown, R. H., \& Cruikshank, D. P. 1997, Ann. Rev. Earth Planet. Sci., 25, 243

Brown, R. H., Cruikshank, D. P., Pendleton, Y., \& Veeder, G. J. 1997, Science, 276, 937 1998, Science, 280, 1430

Cruikshank, D. P., Roush, T. L., Moore, J. M., Sykes, M., Owen, T. C., Bartholomew, M. J., Brown, R. H., \& Tryka, K. A. 1997, in Pluto and Charon, ed. S. A. Stern \& D. J. Tholen (Tucson: Univ. Arizona Press), 221

Cruikshank, D. P., et al. 1998, Icarus, submitted

Davies, J. K., Roush, T. L., Cruikshank, D. P., Bartholomew, M. J., Geballe, T. R., Owen, T., \& de Bergh, C. 1997, Icarus, 127, 238

Davies, J. K., Sykes, M. V., \& Cruikshank, D. P. 1993, Icarus, 102, 166
Grundy, W. M., \& Schmitt, B. 1998, J. Geophys. Res., in press

Hapke, B. 1981, J. Geophys. Res., 86, 3039

Jewitt, D., \& Kalas, P. 1998, ApJ, 499, L103

Lançon, A., \& Rocca-Volmerange, B. 1992, A\&AS, 593

Levison, H. F., \& Duncan, M. J. 1997, Icarus, 127, 13

Luu, J. X., \& Jewitt, D. C. 1996, AJ, 112, 2310 1998, ApJ, 494, L117

Luu, J. X., Jewitt, D. C., \& Cloutis, E. 1994, Icarus, 109, 133

Matthews, K., \& Soifer, B. T. 1994, in Infrared Astronomy with Arrays: The Next Generation, ed. I. McLean (Dordrecht: Kluwer), 239

Tegler, S. C., \& Romanishin, W. 1998, Nature, 392, 49

Wilson, P. D., Sagan, C., \& Thompson, W. R. 1994, Icarus, 107, 288 